\title{
PERCEPÇÃO DE DISCENTES FRENTE AO USO DE ROTEIROS DE ESTUDO EM AULAS PRÁTICAS DE NEUROANATOMIA
}

\author{
PERCEPCIÓN DE DISCENTES FRENTE AL USO DE ROTEIROS DE ESTUDIO EN \\ CLASES PRÁCTICAS DE ANATOMÍA HUMANA Y NEUROANATOMÍA
}

\author{
STUDENTS' PERCEPTION REGARDING THE USE OF STUDY SCRIPTS IN \\ PRACTICAL CLASSES OF NEUROANATOMY
}

\author{
Cristiane Torres Guimarães MENEZES ${ }^{1}$ \\ Edvaldo Xavier da SILVA JUNIOR ${ }^{2}$ \\ Gilberto Santos CERQUEIRA ${ }^{3}$
}

RESUMO: O objetivo do estudo foi analisar a percepção de discentes do curso de fisioterapia, a partir do uso de roteiros didáticos em aulas práticas da disciplina de Neuroanatomia. Este foi do tipo qualitativo, através de relato de experiência, no qual realizouse a análise de conteúdo dos discursos dos partícipes. Foi possível observar que os roteiros lhes facilitaram a compreensão do conceito do componente curricular aqui trabalhado, bem como se apresentou como um método norteador no processo de aprendizagem e foram dinâmicos e facilitadores na apreensão dos conteúdos estudados. Assim, inferimos que o uso de roteiros didáticos em aulas práticas da disciplina de neuroanatomia contribui no processo de ensino-aprendizagem, tornando o discente ativo na construção de seu conhecimento.

PALAVRAS-CHAVE: Anatomia. Manual. Aprendizagem. Ensino. Fisioterapia.

RESUMEN: El objetivo del estudio fue analizar la percepción de discentes, del curso de Fisioterapia, frente al uso de manuales didácticos en clases prácticas de la disciplina de Neuroanatomía. Este fue del tipo cualitativo, a través de relato de experiencia, en el cual se realizó el análisis de contenido de los discursos de los partícipes. Se pudo observar que los manuales les facilitaron la comprensión del concepto del componente curricular aquí trabajado, así como se presentó como un método orientador en el proceso de aprendizaje y fueron dinámicos y facilitadores en la aprehensión de los contenidos estudiados. Así,

\footnotetext{
${ }^{1}$ Universidade de Pernambuco (UPE), Petrolina - PE - Brasil. Graduada em Enfermagem na Universidade de Pernambuco - Campus Petrolina. Durante a graduação, foi monitora de Anatomia Humana e Neuroanatomia por 4 semestres. Participou como voluntária do projeto de monitoria intitulado "Elaboração de roteiros de aulas práticas, nas disciplinas de Anatomia Humana e Neuroanatomia na Universidade de Pernambuco, Campus Petrolina." ORCID: http://orcid.org/0000-0002-7509-3542. E-mail: kristymenezes2006@ hotmail.com

${ }^{2}$ Universidade de Pernambuco (UPE), Petrolina - PE - Brasil. Doutorando do Programa de Engenharia Biomédica da Faculdade de Ciência e Tecnologia (FCT) da Universidade Nova de Lisboa (UNL), o qual faz parte do Grupo de Pesquisa em Hemodinâmica Arterial em cadáver. Mestre em Educação em Ciências pela Universidade Federal do Rio Grande do Sul (UFRGS). ORCID: http://orcid.org/0000-0001-9502-802X. E-mail: edivaldo.junior@upe.br

${ }^{3}$ Universidade Federal do Ceará (UFCE), Fortaleza - CE - Brasil. Doutor em Farmacologia pela Universidade Federal do Ceará. Possui experiência na área de Anatomia Humana, Ciências Morfológicas, Farmacologia, Toxicologia. Possui treinamento em Metodologia ativas: Problem-based learning (PBL) pela University of New Mexico School of Medicine. Possui Aprimoramento em Plastinação pela University of Toledo, Ohio, Estados Unidos. ORCID: http://orcid.org/0000-0001-6717-3772. E-mail: giufarmacia@ hotmail.com
} 
inferimos que el uso de manuales didácticos, en clases prácticas de la disciplina de neuroanatomía, contribuyen en el proceso de enseñanza-aprendizaje, haciendo el discente activo en la construcción de su conocimiento.

PALABRAS CLAVE: Anatomía. Manual. Aprendizaje. Enseñanza. Fisioterapia.

ABSTRACT: The aim of study was to analyze the students' perception of Physiotherapy course, based on the use of didactic scripts in practical classes of Neuroanatomy discipline. It was of qualitative type, through an experience report, in which the content analysis of discourses of participants was carried out. It was possible to observe that scripts facilitated their comprehension of concept of curricular component worked here, as well as presented like guiding method in learning process and were dynamic and facilitators in the apprehension of contents studied. Thus, we infer that use of didactic scripts, in practical classes of Neuroanatomy discipline, contribute in teaching-learning process, making student active in construction of his knowledge.

KEYWORDS: Anatomy. Manual. Learning. Teaching. Physiotherapy.

\section{Introdução}

A anatomia (ana- $=$ através de; - tomia $=$ processo de corte $)$ é a ciência que estuda, macro e microscopicamente, através de cortes, as estruturas do corpo humano e as relações entre as mesmas (DE MENESES, 2010; GRAY, 2012). Assim, designa-se ramo da ciência que estuda a morfologia, a estrutura e a arquitetura do corpo humano (FREITAS et al., 2008; YAMMINE, 2014).

Seu estudo, como disciplina obrigatória de base curricular nos cursos de saúde, tem na graduação a importância de introduzir o conhecimento essencial para a futura compreensão de disciplinas específicas da área, determinando o desempenho do aluno frente aos conteúdos (ARRUDA; SOUSA, 2014).

Tradicionalmente, profissionais e estudantes da área de saúde estudam a referida disciplina através de três maneiras distintas: livros, texto-atlas e cadáveres (INFANTOSI; KLEMT, 2000; COSTA et al., 2012). O uso de cadáveres humanos, mesmo que apenas para demonstração de estruturas anatômicas, é considerado indispensável ao seu processo de ensino-aprendizagem (COSTA et al., 2012; FAZAN, 2011; SANTOS, 2011; FALCÃO et al., 2016; RIBEIRO et al., 2017; OLIVEIRA et al., 2017; SOARES et al., 2018).

Porém, a utilização deste tipo de material para o ensino da anatomia humana tem encontrado entraves que problematizam o seu uso, como o número reduzido de corpos cedidos ao ensino e à pesquisa (SALTARELLI; ROSETH, 2014). Desta forma, frente às 
dificuldades na obtenção de cadáveres, o processo de ensino-aprendizagem torna-se complexo e difícil, forçando o discente e docentes a desenvolverem metodologias de ensino voltadas à memorização das nomenclaturas anatômicas, tornando-a monótona, desestimulante e mecanizada (COSTA et al., 2013; OLIVEIRA et al., 2017).

Outros motivos comprometem o aprendizado dos alunos, como: conteúdo programático extenso; material didático insuficiente para o número de alunos usuários; a vasta terminologia anatômica; o pequeno tamanho de algumas estruturas; preparo inadequado das peças; bem como fatores individuais como, por exemplo, a falta de motivação, atenção e o medo ou receio existente quando alguns discentes se deparam com os cadáveres humanos (BRAZ, 2009; COSTA et al., 2013; REIS et al., 2013; FARIA et al., 2014; FERREIRA, 2008; SOARES et al., 2018).

Dentro do contexto do ensino da anatomia humana e da neuroanatomia, muitas vezes caótico, sua complexidade necessita do apoio especial de recursos didáticos aplicados ao seu ensino, mostrando-se como uma tendência satisfatória e estimulando a participação do aluno como sujeito ativo na busca por novas informações (COSTA et al., 2012; GUIRALDES et al., 1995). A criação e utilização de novas técnicas e materiais para o ensino da disciplina facilita o entendimento da aula teórica e promove um aumento no interesse do aluno pela aula prática (ORLANDO, 2009; SILVA et al., 2012).

Métodos alternativos práticos, como a utilização de observações, experimentação, jogos, tecnologia multimídia, jogos de tabuleiro, laboratórios virtuais em 3D, exames de imagens, peças teatrais, cursos online com laboratório, exercícios práticos formativos, produção de desenhos, redes sociais, elaboração de materiais didáticos por discentes, modelos anatômicos sintéticos, modelos funcionais e roteiros de aulas práticas podem despertar o interesse dos estudantes pelos conteúdos e facilitar a aprendizagem (COSTA et al., 2012; JAFFAR, 2012, 2013; MURAKAMI et al., 2014; ANYANWU, 2014; ARAÚJO JÚNIOR et al., 2014; SILVA JÚNIOR et al., 2014a; SILVA JÚNIOR et al., 2014b; MCNULTY et al., 2015; SHOEPE et al., 2015; ATTARDI; ROGERS, 2015; BARRY et al., 2016; ALSAID; BERTRAND, 2016; HENNESSY et al., 2016; MATHIOWETZ et al., 2016; FALCÃO et al., 2016; RIBEIRO et al., 2017; SILVA et al., 2017; SILVA et al., 2018).

Diante do exposto, buscar métodos que contribuam para a aprendizagem da anatomia humana e neuroanatomia apresenta-se como um grande potencial no processo de ensinoaprendizagem nos cursos de saúde, permitindo que o estudante seja mais participativo e crítico dentro do processo de construção de seu conhecimento. Um destes métodos é o uso de roteiros em aulas práticas dos referidos componentes curriculares, os quais se comprometem 
em auxiliar e orientar o estudante em seu processo de aprendizagem dentro das disciplinas no momento das aulas práticas no laboratório, e fora dele. Porém, a sua análise, diante da construção do conhecimento, não é conhecida na comunidade científica, justificando assim a importância do presente estudo no processo de ensino-aprendizagem dos referidos componentes curriculares a docentes e estudantes.

Assim, o objetivo deste estudo foi analisar a percepção de discentes do curso de fisioterapia frente ao uso de roteiros de aulas práticas empregados nas disciplinas de anatomia humana e neuroanatomia da Universidade de Pernambuco, Campus Petrolina.

\section{Material e métodos}

\section{Método e sujeitos da pesquisa}

O estudo é do tipo qualitativo, o qual evidencia aspectos humanos subjetivos (SILVA; MENEZES, 2001), realizado através do relato de experiência, metodologia que consente a descrição de experiências vividas (GIL, 2002).

O referido tipo de estudo foi escolhido por ser o mais indicado em investigar as percepções e expectativas dos participantes, consentindo a realização da análise e classificação da proposta aplicada, a fim de compreender mais o desempenho dos fatos que abarcam os seus significados do que a constância com que eles acontecem (RICHARDSON, 1999; TOBAR; YALOUR, 2001).

Participaram deste estudo 40 discentes, dos quais 21 foram necessários para atingir os critérios de saturação - conforme Thiry-Cherques (2009) -, do segundo período do curso de Fisioterapia da UPE - Campus Petrolina, cujos roteiros foram aplicados durante as aulas práticas da disciplina de neuroanatomia no Laboratório de Anatomia Humana da referida instituição.

\section{Aspectos éticos e procedimento de coleta dos dados}

Esta pesquisa, atendendo aos procedimentos previstos na resolução $n^{\circ}$. 466/2012 do Conselho Nacional de Saúde, foi aprovada pelo comitê de ética em pesquisa (CEP) da UPE, através do protocolo CAAE: 34051114.8.0000.5207, não havendo conflito de interesses. Os discentes foram informados que seus dados pessoais seriam preservados, e que as informações prestadas não seriam utilizadas para identificá-los. Todos os participantes assinaram o Termo 
de Consentimento Livre e Esclarecido e em nenhum momento foram coagidos ou incitados a alterarem seus conteúdos.

Para analisar a percepção dos discentes, lhes foram entregues, no início de todas as aulas de neuroanatomia prática, um roteiro elaborado por Silva Júnior et al. (2014b) com a seguinte organização: introdução, objetivos, materiais a serem utilizados na aula, conteúdo sobre as estruturas abordadas nos referidos assuntos, questionamentos e referências (Figura $1)$.

Após o término da aplicação dos 16 roteiros, utilizou-se a seguinte pergunta norteadora: "Como o uso de roteiro em aula prática contribuiu no seu processo de aprendizagem na disciplina de Neuroanatomia?", a fim de identificar as sensações e os reais potenciais obtidos pelos partícipes do estudo, permitindo analisar sua percepção de mais um método complementar aos tradicionais, para o referido componente curricular, flexibilizando o seu aprendizado. Cada aluno participante foi representado da seguinte maneira: A1, A2, A3, ..., A20, A21, A22; permitindo o seu anonimato, como previsto nos aspectos éticos.

Figura 1 - Estruturação da elaboração dos roteiros
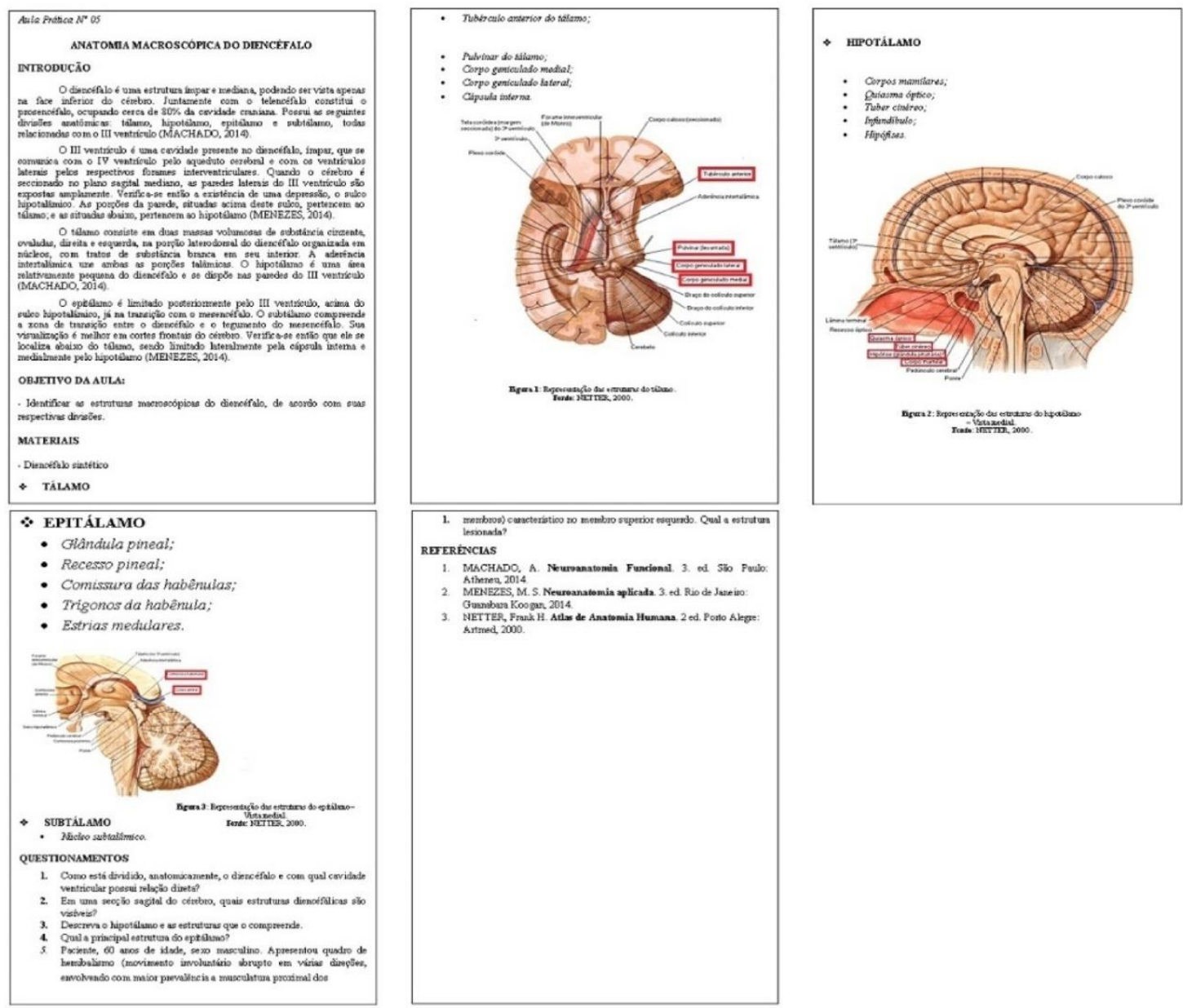

Fonte: Silva Júnior et al. (2014b). 


\section{Análise dos resultados}

Os relatos foram analisados pelo método proposto por Minayo (2004), cuja análise parte em busca dos significados, manifestos e latentes do material coletado por meio dos discursos dos envolvidos no estudo. Para operacionalizar a técnica, seguiu-se suas três etapas. $\mathrm{Na}$ primeira, organizou-se os dados com a transcrição das respostas obtidas, seguida da releitura do material, organizando-o em categorias; após a primeira etapa ordenou-se os dados, realizando leitura extenuante e repetida dos textos, com a detecção dos núcleos de sentido; e por fim, a análise final por meio de triangulação com o referencial teórico e o material coletado.

\section{Resultados e discussão}

Atualmente, com a diminuição da aquisição de material cadavérico, meios alternativos têm sido utilizados com maior frequência e eficiência, como promotores no processo de ensino-aprendizagem da disciplina de neuroanatomia.

Moraes, Schwingel e Silva Júnior (2016) realizaram um levantamento bibliográfico, cujo objetivo era obter informações sobre o uso de modelos anatômicos e de roteiros para aulas práticas de anatomia humana nas instituições de ensino superior. Com isso, perceberam que o uso de modelos anatômicos tem sido utilizado com mais frequência que os roteiros de aulas práticas. Para este último, apenas um trabalho, dentre os 17 analisados, versava sobre a produção de roteiros de aulas práticas para o referido componente curricular, o de Silva e Brito (2013). Desta forma, os pesquisadores enfatizaram a importância de realizar investigações sobre a sua eficiência no processo de ensino e aprendizagem.

Assim, surgiu a presente proposta de estudo, a fim de analisar a percepção dos discentes, após o uso de roteiros em aulas práticas da disciplina de neuroanatomia. Com a análise dos dados, permitiu-se a construção de três categorias empíricas, são elas: percepção do discente sobre o conceito de anatomia humana e neuroanatomia; método pedagógico norteador do processo da aprendizagem; e dinamicidade e lúdico.

\section{Percepção do discente sobre o conceito de anatomia humana e neuroanatomia}

O conceito mais atual de Anatomia foi proposto em 1981, pela American Association of Anatomists (AAA), como sendo uma análise da estrutura biológica, correlacionada com a 
função e com as modulações de estrutura em resposta a fatores temporais, genéticos e ambientais. É atualmente conceituada como a ciência que estuda, macro e microscopicamente, a constituição e o desenvolvimento do corpo humano (DE MENESES, 2010; GRAY, 2012).

De acordo com as respostas dos discentes, percebe-se que eles possuíam noções básicas sobre o conceito de anatomia humana e neuroanatomia, como pode ser observado em seus relatos, transcritos abaixo.

"O estudo de todas as estruturas anatômicas do corpo, desde o sistema mais simples ao mais complexo" (A18).

"Estudo dos constituintes microscópicos do corpo humano e suas funcionalidades, com intuito de conhecer essas estruturas e suas atuações na sobrevivência do ser humano" (A8).

"Estudo das estruturas do corpo humano (músculos, ossos, articulações) e do SNC e SNP" (A3).

"A ciência que estuda tudo o que constitui o corpo, suas funções, a composição em si para compreender o corpo humano" (A20).

"A ciência que estuda a morfologia e o funcionamento de todas as estruturas correlacionadas ao cérebro e ao SN. É bem interessante estudar o funcionamento do nosso corpo" (A1).

"Estudo das diferentes estruturas do corpo humano e o seu funcionamento" (A6).

"Estudo do sistema nervoso como um todo, assim como do corpo humano e suas estruturas" (A10).

Fontelles et al. (2006) elaboraram um livro/dicionário texto com recursos informacionais de software (CD-ROM) contendo um banco de dados extenso de termos anatômicos com os seus respectivos conceitos, possibilitando a consulta e a divulgação do conhecimento acerca da anatomia humana básica. Com isso, os pesquisadores puderam perceber que este material educacional interativo contribuiu, diretamente, no aprendizado e na formação profissional dos estudantes de graduação.

Schlemmer (2014) desenvolveu um estudo em que seu objetivo era compreender como poderiam ser desenvolvidos os processos de ensino e de aprendizagem de conceitos de anatomia humana na perspectiva do immersive learning, utilizando metaversos. Com isso, pode concluir que os sistemas modelados em 3D facilitaram a atribuição de significado a conceitos e processos vinculados aos diferentes sistemas, compondo um ambiente híbrido e propiciando um maior envolvimento dos discentes nos conceitos.

Os achados dos autores acima puderam ser corroborados com o presente estudo, uma vez que foi constatado que um método alternativo ao ensino tradicional da anatomia facilita o aprendizado e a compreensão de seus conceitos. Informação importante para o conhecimento 
de estruturas mais complexas dentro de seu conteúdo, facilitando assim o processo de aprendizagem em neuroanatomia.

\section{Método pedagógico norteador do processo de aprendizagem}

Segundo Fontelles et al. (2006) e Da Silva et al. (2013), o processo de ensinoaprendizagem da disciplina de anatomia humana apresenta-se complexo e difícil, uma vez que a memorização de estruturas infindáveis, e com nomenclaturas bastante complexas pela especificidade, torna a atividade monótona e, por vezes, desestimula o interesse da maioria dos alunos, quando não ministrada de maneira dinâmica, corroborando com Reis et al. (2013) e Faria et al. (2014), estendendo essa problemática à disciplina de neuroanatomia, também.

Prigol e Giannotti (2008) e Murakami et al. (2014) afirmam que a ausência de métodos práticos nas aulas de anatomia, bem como a inaptidão dos professores, encontram-se relacionados com as dificuldades na assimilação dos conteúdos com que os alunos se deparam. No que discerne a Neuroanatomia, o pequeno tamanho das estruturas, preparações cadavéricas inadequadas e estruturas que não são visíveis macroscopicamente, comprometem o entendimento e a compreensão dos conteúdos trabalhados (FALCÃO et al., 2016).

Para Falcão et al. (2016), Silva et al. (2017), Ribeiro et al. (2017), Oliveira et al. (2017), Nascimento et al. (2018), Soares et al. (2018) e Silva et al. (2018), métodos alternativos são recursos facilitadores na compreensão e fixação de temas da Neuroanatomia, corroborando com os autores Silva et al. (2012), os quais ainda complementam que a mediação no estudo do referido componente curricular contribui neste processo.

Os discentes participantes do presente estudo relataram que o método alternativo proposto lhes auxiliou na apreensão das nomenclaturas utilizadas, resultados expressos nos relatos abaixo, sendo uma alternativa complementar, como abordado pelos autores supracitados.

\footnotetext{
"Este tipo de didática facilita a aprendizagem deixando-a mais apreciável e não tão monótona pois o conteúdo é extenso e difícil" (A6).

"Facilita o aprendizado, uma vez que, temos com esse roteiro, o caminho que se deve tomar para a fixação dos mais variados conteúdos" (A17).

"Norteiam melhor o aluno para assimilar e compreender o conteúdo" (A21).

"Facilitam o entendimento e fixação do conteúdo, pois o aluno se preocupa mais em prestar atenção do que escrever o conteúdo" (A12).
} 
"Essencial e indispensável, pois melhora a assimilação da aula ministrada" (A7).

"Muito proveitoso, pois concilia a teoria e a prática, reunindo as informações mais relevantes a serem exploradas" (A14).

Krapas et al. (1997) e Cavalcante e Silva (2008) referem-se a modelos pedagógicos como métodos alternativos de ensino, sendo uma representação simplificada de uma ideia, objeto, evento, processo ou sistema que se constitua em objeto de estudo, onde o mesmo favorece o processo de aprendizagem da anatomia e contribui na relação da teoria com a prática. A ideia dos referidos autores pode ser observada, também, nos estudos de Falcão et al. (2016), Silva et al. (2017), Ribeiro et al. (2017) e Nascimento et al. (2018).

Semelhantemente ao que foi relatado pelos autores acima, os participantes do presente estudo mencionaram que métodos alternativos no ensino da Neuroanatomia cooperam e direcionam para a associação do conteúdo teórico. Assim, os roteiros para aulas práticas, além de facilitar a apreensão dos conteúdos vivenciados na disciplina, apresentam-se, também, como um método lúdico que envolve o discente em todo o processo, tornando a atividade prazerosa dentro de toda a metodologia aplicada.

\section{Dinamicidade e lúdico}

Por ser uma ciência que estuda a constituição e o funcionamento do corpo humano, a anatomia humana possui uma grande quantidade de estruturas, as quais o discente necessita apreendê-las, muitas vezes, em um espaço de tempo muito curto, prejudicando assim o seu processo de aprendizado. Além disso, uma frequência maior de aulas teóricas do que práticas faz com que os discentes se desestimulem e não deem o devido interesse à disciplina, ficando a cargo do docente abordar o conteúdo de maneira dinâmica para prender a atenção.

Autores como Miranda (2001), Schultz et al. (2005), Brito et al. (2005) e Santos et al. (2008) mencionam o uso de atividades lúdicas como métodos essenciais e eficientes na construção do aprendizado nas diferentes áreas da saúde, principalmente em temas relacionados à anatomia humana.

Fornaziero e Gil (2003), Fornaziero et al. (2010), Araújo et al. (2014), Yammine (2014) e Oliveira et al. (2017) afirmam que metodologias inovadoras auxiliam a dinamizar as aulas e o estudo da anatomia para os estudantes de graduação, inserindo-os no processo de ensino e aprendizagem. Semelhantemente, Silva et al. (2012) relataram que a diversidade de materiais pedagógicos facilita o aprendizado, tornando as aulas práticas mais dinâmicas e produtivas, propiciando uma maior assimilação e entendimento do conteúdo ministrado. 
O uso de roteiros em aulas práticas, aplicado neste estudo, atingiu resultados semelhantes aos dos autores acima, uma vez que é uma metodologia alternativa e ativa em que o estudante é direcionado à construção de seu aprendizado, bem como inovadora a nível da comunidade científica.

\footnotetext{
"Os roteiros tornaram a aula mais dinâmica e facilitou a aprendizagem" (A8).

"É um método diferente, mais dinâmico, faz com que haja uma maior absorção dos conteúdos e melhor fixação" (A21).

"Mostra através de outras literaturas o assunto abordado, enquanto se aprende de uma forma mais lúdica" (A1).

"É bem lúdico para professor e aluno, além de facilitar o aprendizado, deixando o assunto mais envolvente" (A5).

"Torna a aula mais interativa e compreensiva, pois todos precisam absorver o conhecimento, trazendo resultados mais eficientes na aprendizagem" (A20).

"Muito útil para fixação do conteúdo e aprendizagem de forma mais dinâmica" (A21).
}

Através dessas declarações pode ser observado que a utilização do método aqui empregado, como material alternativo, facilita o entendimento da aula teórica e aumenta o interesse dos estudantes pelo conteúdo, além de se tornar uma alternativa estimuladora no seu processo de aprendizagem, como apresentado por Ribeiro et al. (2018) ao utilizar um outro método complementar, os jogos de tabuleiro.

Segundo Santos (1999), o lúdico é importante em todas as faixas de idade, e não pode ser visto apenas como diversão, já que facilita o processo de construção do conhecimento. Sendo assim, torna-se de fundamental importância a dinamização das aulas, tendo em vista a motivação dos discentes.

Schultz et al. (2005) afirma que as atividades lúdicas são uma forma moderna de ensinar em sala de aula, representando uma maneira prazerosa de trabalhar as dificuldades dos estudantes, promovendo a aquisição do conhecimento.

Nesse sentido, o docente passa a atuar como um mediador do processo de ensinoaprendizagem, deixando de ser o detentor de todo o conhecimento, agindo de forma inovadora, auxiliando os estudantes na busca de soluções para os seus conflitos cognitivos, como afirma Nascimento et al. (2018), sendo um caminho alternativo para o aprendizado. 


\section{Considerações finais}

Hoje em dia, trabalhar métodos alternativos na disciplina de neuroanatomia apresentase como uma opção diante da falta de material cadavérico, sem que haja comprometimento do processo de ensino-aprendizagem, tanto para o docente quanto para o discente.

Desta forma, os roteiros de aulas práticas apresentaram-se como sendo mais um método complementar, alternativo e inovador, já que não há nenhum material deste tipo aplicado, analisado e com seus resultados divulgados na comunidade científica. Assim, concluímos que o seu uso, durante aulas práticas de neuroanatomia, contribui para a compreensão de conceitos e nomenclaturas; direcionam os discentes para a fixação de sua aprendizagem; dinamizam as aulas; e facilitam - consequentemente - o ensino-aprendizado.

Assim, apresentam-se como um método que conduz o aluno à construção de seu próprio conhecimento, permitindo-lhe deixar de ser um agente passivo para ser ativo em seu processo de aprendizagem. Enfatizamos ainda que este é um método complementar, pois jamais o cadáver poderá ser substituído, sendo este a ferramenta mais eficaz no processo de ensino e aprendizagem da Anatomia Humana, bem como da Neuroanatomia.

AGRADECIMENTOS: Os pesquisadores agradecem a todos os estudantes que se disponibilizaram a participar do estudo, a fim de aprimorar o processo de ensinoaprendizagem da disciplina de Neuroanatomia e a toda a equipe do Laboratório de Ensino e Pesquisa em Anatomia Humana (LABEPAH), da Universidade de Pernambuco - Campus Petrolina, pelo apoio na aplicação das atividades deste estudo.

\section{REFERÊNCIAS}

ALSAID, B.; BERTRAND, M. Student's memorization of anatomy, influence of drawing. Morphologie, v.100, n.328, p.02-06, 2016.

ANYANWU, E. G. Anatomy adventure: a board game for enhancing understanding of anatomy. Anatomical Science Education, v. 7, n. 2, p. 153-160, 2014.

ARAÚJO JÚNIOR, J. P.; GALVÃO, G. A. S.; MAREGA, P.; BAPTISTA, J. S.; BEBER, E. H.; SEYFERT, C. E. Desafio anatômico: uma metodologia capaz de auxiliar no aprendizado de anatomia humana. Revista da Faculdade de Medicina de Ribeirão Preto e do Hospital das Clínicas da FMRP, v. 47, n. 1, p. 62-68, 2014.

ARRUDA, R. M.; SOUSA, C. R. A. Aproveitamento Teórico-Prático da Disciplina Anatomia Humana do Curso de Fisioterapia. Revista Brasileira de Educação Médica, v. 38, n. 1, p. 65-71, 2014. 
ATTARDI, S. F.; ROGERS, K. A. Design and implementation of an online systemic human anatomy course with laboratory. Anatomical Sciences Education, v. 8, n. 1, p. 53-62, 2015.

BARRY, D. S.; TIERNEY, P.; O'KEEFFE, G. Anatomy education for the YouTube generation. Anatomical Sciences Education, v. 9, n. 1, p. 90-96, 2016.

BRAZ, P. R. P. Método didático aplicado ao ensino da anatomia humana. Anuário da produção acadêmica docente, v. 3, n. 4, p. 303-310, 2009.

BRITO, S. R.; SANTOS, T. L. T.; DA SILVA, A. S.; COSTA, K.; FAVERO, E. L. Automatizado à mediação da aprendizagem baseada em experimentos. RENOTE, v. 3, n. 2, p. 1-11, 2005. Disponível em:

http://www.seer.ufrgs.br/index.php/renote/article/view/14033/7924. Acesso em: 10 abr. 2018.

CAVALCANTE, D. D.; SILVA, A. F. A. Modelos didáticos e professores: concepções de ensino-aprendizagem e experimentações. In: XIV Encontro Nacional de Ensino de Química, 2008, Curitiba. Anais..., Paraná, UFPR, 2008. Disponível em:

http://www.quimica.ufpr.br/eduquim/eneq2008/resumos/R0519-1.pdf. Acesso em: 10 abr. 2018.

COSTA, B. D. B.; BARRETO, S. D.; VERAS JÚNIOR, E. L.; VIEIRA, G. O.; LUCENA, E. E. S. Corpo humano real e fascinante: a extensão universitária como um elo integrador entre o ensino médio/profissionalizante e o superior. Extendere, v. 2, n. 1, 2013.

COSTA, G. B. F.; COSTA, G. B. F.; LINS, C. C. F. A. O Cadáver no Ensino da Anatomia Humana: uma Visão Metodológica e Bioética. Revista Brasileira de Educação Médica, Rio de Janeiro, v. 33, n. 6, p. 369-373, 2012.

DA SILVA, C. K.; SANTANA, A. O.; DE MORAES, A. S. R. Quality and language of learning objects used in the teaching of human anatomy. International Journal of Morphology., v. 31, p. 455-460, 2013.

DE MENESES, R. D. B. Morfologia da morfologia humana. Revista Ciencias de La Salud, v. 8, n. 01, p. 87-93, 2010.

FALCÃO, J. G. B.; SANDES, A. A. G.; RODRIGUES, B. R.; NASCIMENTO, I. Y. M.; SCHWINGEL, P. A.; SILVA JÚNIOR, E. X. Neuroanatomical structures manufacture: an alternative in the practical teaching of neuroanatomy. Journal of Morphological Science, v. 33 , n. 1, p. 17-21, 2016.

FARIA, J. W. V.; FIGUEIREDO, E. G.; BRITO, D. R.; TEIXEIRA, M. J. A evolução histórica do ensino da Neuroanatomia. Revista de Medicina, v. 93, n. 4, 146-150, 2014.

FAZAN, V. P. S. Métodos de ensino em anatomia: dissecção versus prossecção. Revista de Divulgação Científica da Sociedade Brasileira de Anatomia, Ano 2, v. 1, 2011.

FERREIRA, T. A. Estudo de neurofisiologia associados com modelos tridimensionais construídos durante o aprendizado. Bioscience Journal, v. 24, n. 1, p. 98-103, 2008. 
FONTELLES, M. P.; CARVALHO, R. M.; PEREIRA, N.; JORGE, S. C.; MAIA, M. F. Dicionário de estruturas e termos anatômicos: versão bilíngue Português/inglês empregando multimídia em cd-rom. Revista Paraense de Medicina, v. 20, n. 2, p. 7-12, 2006.

FORNAZIERO, C. C.; GIL, C. R. R. Novas Tecnologias Aplicadas ao Ensino da Anatomia Humana. Revista Brasileira de Educação Médica, v. 27, n. 2, p. 141-146, 2003.

FORNAZIERO, C. C.; GORDAN, P. A.; CARVALHO, M. A. V.; ARAÚJO, J. C.; AQUINO, J. C. B. O Ensino da Anatomia: Integração do Corpo Humano e Meio Ambiente. Revista Brasileira de Educação Médica, v. 34, n. 2, p. 290-297, 2010.

FREITAS, I. C. M. Pesquisa Qualitativa em Saúde: um olhar inovador sobre a produção do conhecimento científico. Revista Baiana de Saúde Pública. v. 35, n. 4, p. 1001-1012, 2011.

FREITAS, L. A. M.; BARROSO, H. F. D.; RODRIGUES, H. G.; AVERSI-FERREIRA, T. A. Construção de modelos embriológicos com material reciclável para uso didático.

Bioscience Journal, v. 24, n. 1, p. 91-97, 2008.

GRAY, H.; GOSS, C. M. Anatomia. 29. ed. Rio de Janeiro: Guanabara Koogan, 2012.

GUIRALDES, C. H.; ODDÓ, A. H.; ORTEGA, F. X.; OYARZO, M. P. Métodos computacionales y gráficos de apoyo al aprendizaje de la anatomia humana: vision de los estudiantes. Revista Chilena de Anatomia, v. 13, n. 1, p. 67-71, 1995.

HENNESSY, C. M.; KIRKPATRICK, E.; SMITH, C. F.; BORDER, S. Social media and anatomy education: using twitter to enhance the student learning experience in anatomy. Anatomical Sciences Education, v. 9, n. 6, p. 505-515, 2016.

INFANTOSI, A. F. C.; KLEMT, A. Visualização 3D da dissecação do crânio humano. Revista Brasileira de Engenharia Biomédica, v. 16, n. 1, p. 21-37, 2000.

JAFFAR, A.A. YouTube: An Emerging Tool in Anatomy Education. Anatomical Science Educational, v. 5, n. 3, p. 158-164, 2012.

JAFFAR, A.A. Exploring the Use of a Facebook Page in Anatomy Education. Anatomical Science Educational, v. 7, n. 3, p. 199-208, 2013.

KRAPAS, S.; QUEIROZ, G.; COLINVAUX, D.; FRANCO, S. Modelos: Uma análise de sentidos na literatura de pesquisa em ensino de ciências. Investigações em Ensino de Ciências. v. 2, n. 3, p. 185-205, 1997.

MATHIOWETZ, V.; YU, C. H.; QUAKE-RAPP, C. Comparison of a gross anatomy laboratory to online anatomy software for teaching anatomy. Anatomical Sciences Education, v. 9, n. 1, p. 52-59, 2016.

MCNULTY, J. A.; ESPIRITU, B. R.; HOYT, A. E.; ENSMINGER, D. C.; CHANDRASEKHAR, A. J. Associations between formative practice quizzes and summative examination outcomes in a medical anatomy course. Anatomical Sciences Education, v. 8, n. 1, p. 37-44, 2015. 
MINAYO, M. C. S. O desafio do conhecimento: pesquisa qualitativa em saúde. 8. ed. São Paulo: Hucitec, 2004.

MIRANDA, S. No fascínio do jogo: a alegria de aprender. Ciência Hoje, v. 28, n. 168, p. 6466, 2001.

MORAES, G.N.B.; SCHWINGEL, P.A.; SILVA JÚNIOR, E.X. Uso de roteiros didáticos e modelos anatômicos, alternativos, no ensino-aprendizagem nas aulas práticas de anatomia humana. Revista Ibero-Americana de Estudos em Educação, v. 11, n.1, 2016. Disponível em: https://periodicos.fclar.unesp.br/iberoamericana/article/view/7305/5709. Acesso em: 18 jun. 2018.

MURAKAMI, T.; TAJIKA, Y.; UENO, H.; AWATA, S.; HIRASAWA, S.; SUGIMOTO, M.; KOMINATO, Y.; TSUSHIMA, Y.; ENDO, K.; YORIFUJI, H. An integrated teaching method of gross anatomy and computed tomography radiology. Anatomical Sciences Education, v. 7, n. 6, p. 438-449, 2014.

NASCIMENTO, I. Y. M. et al. Hands-on': an active experience in manufacturing parasitized neuroanatomical models as a teaching and learning tool. International Journal of Development Research, v. 8, n. 5, p. 20377-20381, 2018. Disponível em: http://www.journalijdr.com/sites/default/files/issue-pdf/12732.pdf. Acesso em: 18 jun. 2018.

OLIVEIRA, P. S. et al. The teaching of human anatomy in Brazil: the reality of Northeast Brazil. International Journal of Anatomy and Research, v. 5, n. 4.2, p. 4623-4629, 2017.

ORLANDO, T. C. Planejamento, montagem e aplicação de modelos didáticos para abordagem de biologia celular e molecular no ensino médio por graduandos de ciências biológicas. Revista Brasileira de Ensino de Bioquímica e Biologia Molecular, v. 1, n. 1, p. $1-17,2009$.

PRIGOL, S.; GIANNOTTI, S. M. A importância da utilização de práticas no processo de ensino-aprendizagem de ciências naturais enfocando a morfologia da flor. In: I Simpósio Nacional de Educação - XX Semana da Pedagogia, 2008, Cascavel. Anais..., Paraná, Unioeste, 2008. p.1-12. Disponível em:

http://www.unioeste.br/cursos/cascavel/pedagogia/eventos/2008/1/artigo\%2033.pdf. Acesso em: 19 abr. 2018.

REIS, C.; MARTINS, M. M.; MENDES, R. A. S.; GONSALVES, L. B.; FILHO, H. C. S.; MORAIS, M. R. Avaliação da percepção de discentes do curso médico acerca do estudo anatômico. Revista Brasileira de Educação Médica, v. 37, n. 03, p. 350-358, 2013.

RIBEIRO, F. S. et al. Neurogame: an alternative and complementary method in the teaching and learning process of neuroanatomy. International Journal of Research \& Methodolgy in Social Science, v. 3, n. 1, p. 62-71, 2017. Disponível em: https://drive.google.com/file/d/0B2B2CwoNDenGZVQwYWxKN1VBNTg/view. Acesso em: 18 jun. 2018.

RICHARDSON, R. J. Pesquisa social: métodos e técnicas. São Paulo: Atlas, 1999. 
SALTARELLI, A. J.; ROSETH, C. J. Effects of synchronicity and belongingness on face-toface and computer-mediated constructive controversy. Journal of Educational Psychology, v. 106, n. 4, p. 946-960, 2014.

SANTOS, M. C. et al. A Anatomia humana para a enfermagem: diálogos interdisciplinares no currículo. Revista de Educação, São Paulo, v. 7, n. 15, p. 181-190, 2011.

SANTOS, S. M. P. O Lúdico na Formação do Educador. Petrópolis: Vozes, 1999.

SANTOS, V. P. A.; SILVA, K. S.; NOVAIS, R. M.; MARCONDES, M. E. R. Modelos didáticos revelados nos discursos dos professores em formação. In: XIV Encontro Nacional de Ensino de Química - XIV ENEQ, 2008, São Paulo. Anais..., São Paulo, 2008. Disponível em: http://www.cienciamao.usp.br/dados/eneq/_modelosdidaticosrevelado.trabalho.pdf. Acesso em: 19 abr. 2018.

SCHLEMMER, E. Laboratórios digitais virtuais em 3d: anatomia humana em metaverso, uma proposta em immersive learning. Revista e-Curriculum, São Paulo, v. 12, n. 3 p. 2119-2157, 2014.

SCHULTZ, E. S.; MULLER, C.; CÔRREA, S. M. M. Laboratório de aprendizagem: o lúdico nas séries iniciais. 2005. Disponível em: http://coperves.ufsm.br/prograd/downloads/File/Laboratóriodeaprendizagem.pdf. Acesso em: 20 abr. 2018.

SHOEPE, T. C.; CAVEDON, D. K.; DERIAN, J. M.; LEVY, C. S.; MORALES, A. The ATLAS project: the effects of a constructionist digital laboratory Project on undergraduate laboratory performance. Anatomical Science Education, v. 8, n. 1, p. 12-20, 2015.

SILVA, C. F. et al. Análise da satisfação do uso de exames de imagem, como proposta complementar, no processo de ensino e aprendizagem da anatomia sistêmica. Revista Saúde \& Ciência Online, v. 7, n. 1, p. 35-44, 2018. Disponível em: http://www.ufcg.edu.br/revistasaudeeciencia/index.php/RSC-UFCG/article/view/527/326. Acesso em: 18 jun. 2018.

SILVA JÚNIOR, E. X. et al. Elaboração de modelos anatômicos alternativos para o ensinoaprendizagem da disciplina de neuroanatomia humana, a partir de material de baixo custo. In: I Congresso Nacional de Educação - I CONEDU, 2014, Campina Grande. Anais [...], Paraíba: Realize, v. 1, n. 1, p. 1-05, 2014a. Disponível em: http://www.editorarealize.com.br/revistas/conedu/trabalhos/Modalidade_1datahora_14_07_ 2014_19_51_54_idinscrito_3160_eec18973fabcaf6b30f5b526540557cc.pdf. Acesso em: 18 jun. 2018.

SILVA JÚNIOR, E. X. et al. Elaboração de roteiros para o ensino-aprendizagem nas aulas práticas das disciplinas de anatomia humana e neuroanatomia. In: CONEDU, 1., 2014, Campina Grande. Anais [...] Paraíba: Realize, p. 1-5, 2014b. Disponível em: http://www.editorarealize.com.br/revistas/conedu/trabalhos/Modalidade_1datahora_15_07_ 2014_22_59_18_idinscrito_3160_6ad18d13d81622ec0227b39630b0b40a.pdf. Acesso em: 18 jun. 2018. 
SILVA, K. R. S.; BRITO, V. C. Manual de aula prática para o ensino de anatomia humana. In: XIII Jornada de Ensino, Pesquisa e Extensão - XIII JEPEX - UFRPE, 2013, Recife. Anais [...], Pernambuco, 2013. p. 1-3. Disponível em: http://www.eventosufrpe.com.br/2013/cd/resumos/R0128-1.pdf. Acesso em: 10 jun. 2018.

SILVA, M. S. L.; MACHADO, H. A.; BIAZUSSI, H. M. Produção de Material Didático Alternativo para Aula Prática de Anatomia Humana. In: Congresso Norte Nordeste de Pesquisa e Inovação, 2012, Palmas. Anais [...], Tocantins, 2012. p. 1-7. Disponível em: http://propi.ifto.edu.br/ocs/index.php/connepi/vii/paper/viewFile/4211/1560. Acesso em: 20 mar. 2018.

SILVA, Y. A. et al. Confecção de modelo neuroanatômico funcional como alternativa de ensino e aprendizagem para a disciplina de neuroanatomia. Revista Ibero-Americana de Estudos em Educação, Araraquara, v. 12, n. 3, p. 1674-1688, 2017. Disponível em: http://dx.doi.org/10.21723/riaee.v12.n.3.8502. Acesso em: 18 jun. 2018.

SOARES, B. O. et al. Are corpses the best method for practical anatomy assessment, according to health course students? Journal of Morphological Science, v. 35, n. 1, p. $70-79$ 2018.

THIRY-CHERQUES, H. R. Saturação em pesquisa qualitativa: estimativa empírica de dimensionamento. Revista PMKT. v. 3, n. 4, p. 20-27, 2009.

TOBAR, F.; YALOUR, M. R. Como fazer teses em saúde pública: conselhos e ideias para formular projetos e redigir teses e informes de pesquisa. Rio de Janeiro: Fiocruz, 2001.

YAMMINE, K. Evidence-based Anatomy. Clinical Anatomy, v. 27, n. 6, p. 847-852, 2014. Disponível em: https://onlinelibrary.wiley.com/doi/epdf/10.1002/ca.22397. Acesso em: 18 jun. 2018.

\section{Como citar este artigo:}

MENEZES, Cristiane Torres Guimarães, SILVA JUNIOR, Edvaldo Xavier; CERQUEIRA, Gilberto Santos. Percepção de discentes frente ao uso de roteiros de estudo em aulas práticas de neuroanatomia. Revista Ibero-Americana de Estudos em Educação, Araraquara, v. 14, n. $\quad 4$, p. 2244-2259, out./dez., out./dez., $2019 . \quad$ e-ISSN: 1982-5587. DOI: https://doi.org/10.21723/riaee.v14i3.11496

Data de Submissão: $26 / 06 / 2018$

Revisões requeridas: 10/11/2018

Aceite em: 18/04/2019

Publicado em: 02/09/2019 